\title{
Fe nanoparticle entrained in tubular carbon nanofiber as an effective electrode material for metal-air batteries: a fundamental reason
}

Taegon Kim ${ }^{\mathrm{a}}$, Yuzo Ohata ${ }^{\mathrm{a}}$, Jandee Kim ${ }^{\mathrm{a}}$, Choong Kyun Rhee ${ }^{\mathrm{b}, *}$, Jin Miyawaki ${ }^{\mathrm{a}}$, Seong-Ho Yoon $^{\mathrm{a}, *}$

${ }^{a}$ Institute for Materials Chemistry and Engineering, Kyushu University, Fukuoka, 816-8580, Japan

${ }^{b}$ Department of Chemistry, Chungnam National University, Daejeon, 305-764, Korea

\begin{abstract}
Fe nanoparticle entrained in tubular carbon nanofiber (TCNF) as an effective electrode material for metal-air batteries was successfully prepared. TEM and STM investigations revealed that during impregnation Fe precursor ions penetrated into TCNF tubes through factures between the structural units of TCNF, which were absent in commercial carbon nanotube (CCNT). Thus the location of Fe nanoparticles depended on carbon materials: they were in the TCNF tubes and on the surfaces of CCNT. During repeated voltammetric cycles, the Fe nanoparticles in the TCNF tubes evolved to elongated oxygen-lean Fe nanoparticles, while those on CCNT did to oxygen-rich Fe oxide dendrites. Voltammograms of the modified Fe nanoparticles revealed that a higher overpotential was needed for dendrite formation. On the other hand, impedance spectroscopy also disclosed that the mass transfer of soluble $\mathrm{Fe}$ species was much faster in the one-dimensional TCNF tubes than in the three-dimensional open spaces between individual CCNTs. Thus, a low overpotential of the redox process of the elongated Fe nanoparticles and the fast mass transfer of soluble Fe species in the TCNF tubes resulted in an increased amount of Fe involved in charging/discharging process, which accounted for the higher efficiency of Fe nanoparticles on TCNF in Fe-air battery system.
\end{abstract}

\section{Introduction}

Metal-air battery is an attractive power source applicable to electric vehicles because of its

* Corresponding authors:

TEL: +8192 5837959; FAX: +81925837897

E-mail address: yoon@cm.kyushu-u.ac.jp (S. -H. Yoon)

TEL:+8242 8215483; FAX: +82428218896

E-mail address: ckrhee@cnu.ac.kr(C.K.Rhee) 
high energy density, low cost, and low environmental load [1-5]. Lithium-air batteries have drawn attention due to their improved gravimetric energy storage density [1,5]. However, safety concerns associated with lithium require several precautions, such as employing a nonaqueous electrolyte [6]. Promising alternatives are iron- and zinc-air batteries using alkaline electrolytes, which are advantageous in terms of safety, cost, and environmental load [3]. However, these batteries have disadvantages to be solved before they are commercially available, such as dendrite formation shortening the cycle life of metal-air battery [7-9].

Many efforts have been made to overcome these hurdles toward practical $\mathrm{Fe}$-air or $\mathrm{Zn}$-air batteries [10-17]. Two major concerns in such efforts are hydrogen evolution causing water electrolysis to increase internal pressure of batteries $[5,6,17,18]$ and dendrite formation during repeated charge/discharge processes. To provide solutions to these problems, alloying iron or zinc with a second metal (such as nickel and indium) and coating the alloys with other materials (such as lithium boron oxide and neodymium) has been applied to increase the overpotential of hydrogen evolution $[10,12,14]$. However, problems associated with dendrite formation still remain unsolved because of uncontrollable and irregular dissolution/deposition of iron or zinc metal in alkaline electrolytes. Solid or gel state polymer electrolytes and ionic liquids were proposed as alternatives to avoid dendrite formation in aqueous electrolyte [1,5,19-22]. Another approach is addition of phosphoric acid or citric acid to electrolytes as corrosion inhibitors to increase the stability of iron and zinc $[11,16,17]$. However, excluding lithium-air batteries, the use of these alternatives in iron- and zinc-air batteries remains in its infancy.

Our group investigated $\mathrm{Fe}_{2} \mathrm{O}_{3}$ nanoparticles on various carbon materials for iron-air battery system [23-29]. In an iron-air battery system, $\mathrm{Fe}_{2} \mathrm{O}_{3}$ nanoparticles on tubular carbon nanofiber (TCNF) support was more efficient than other carbon materials such as acetylene black, graphite, and other carbon nanofibers by a factor of $2 \sim 3[24,26,29,30]$. However, the reason for the higher efficiency of TCNF has been remained unclear.

This report presents a fundamental reason for the higher efficiency of Fe nanoparticles loaded in the hollow tubes of TCNF referring to a commercial CNT (CCNT) having a tubular structure of a diameter similar to that of the investigated TCNF. The fundamental reason for the enhancement of TCNF is the existence of fractures (termed "nano-gates") between structural basic units of specially prepared TCNF [31,32], which allow Fe precursor ions to penetrate into TCNF tubes. During an electrochemical treatment, Fe nanoparticles in TCNF tubes evolve to oxygen-lean elongated Fe nanoparticles, while those on the surfaces of CCNT do to oxygen-rich Fe oxide dendrites. The particular difference is related to distinguishing 
electrochemical behavior, which in turn accounts for the higher efficiency of Fe nanoparticles impregnated on TCNF in our previous works.

\section{Experimental}

\subsection{Sample preparation}

Two carbon materials were employed. One was TCNF prepared from a mixture of CO and $\mathrm{H}_{2}$ gas $(1: 1,200 \mathrm{~mL} / \mathrm{min})$ over $\mathrm{Fe} / \mathrm{Ni}(6 / 4, \mathrm{w} / \mathrm{w})$ alloy catalysts at $685^{\circ} \mathrm{C}$ for $1 \mathrm{~h}$ (as described in ref. [31-33]). The other carbon material was a commercial carbon nanotube (CCNT), supplied by JEIO Co., Ltd., Korea. The choice of the specific CCNT as a reference material was based on that among commercially available tubular carbon materials, the diameter of the chosen CCNT was closest to the diameter of TCNF to be compared in this work (See below.). To enhance the structural differences of TCNF and CCNT, they were graphitized at $2800^{\circ} \mathrm{C}$ under an Ar atmosphere for $10 \mathrm{~min}$.

Fe nanoparticles were loaded on TCNF and CCNT using an impregnation method. An aqueous solution of $\mathrm{FeCl}_{3} \cdot 6 \mathrm{H}_{2} \mathrm{O}$ (Wako Pure Chemical Co., Ltd., Japan) with dispersed TCNF or CCNT were stirred and dried for 3 days at $40^{\circ} \mathrm{C}$ to ensure complete penetration of Fe precursor ions into the tubes of TCNF and CCNT, and the residuals after drying were treated at $500^{\circ} \mathrm{C}$ for $1 \mathrm{~h}$ under an $\mathrm{Ar}$ atmosphere to reduce the impregnated Fe precursor ions to metallic Fe and to remove chlorides.

\subsection{Characterizations}

Crystallographic phases of loaded $\mathrm{Fe}$ nanoparticles on carbon materials were identified using X-ray diffraction (XRD, Rigaku Ultima III, CuKa target). The loaded amounts of Fe nanoparticles were estimated using thermogravimetric analysis (TGA, STA7000 series, Seiko Instruments Inc., Japan). Visual observation of TCNF and CCNT with and without Fe nanoparticles was performed using a transmission electron microscope (TEM, JEM-2010F, JEOL Ltd., Japan) with an acceleration voltage of $200 \mathrm{keV}$. After electrochemical treatment, Fe-loaded carbon materials were collected for visual inspection by dissolving with a mixture of N-methyl-2-pyrrolidinone (NMP, Wako Pure Chemical Co., Ltd., Japan) and acetone. Simultaneously, elemental analysis was carried out using energy-dispersive X-ray spectroscopy associated with TEM (TEM-EDX , JEM-2010F, JEOL Ltd., Japan). Also, the exterior surfaces of TCNF and CCNT were observed using scanning tunneling microscopy (STM, Nanoscope IIIa, Digital Instrument, USA). STM images were acquired under a constant current mode using tunneling currents in the range of $0.2 \sim 1.0 \mathrm{nA}$, bias voltage in 
the range of $0.1 \sim 1 \mathrm{~V}$, and scan frequencies of $1 \sim 2 \mathrm{~Hz}$. A few amounts of TCNF and CCNT were suspended in methanol, and then dropped onto the freshly exfoliated surface of highly ordered pyrolytic graphite (HOPG).

\subsection{Electrochemical measurements}

For electrochemical measurements, the electrodes were prepared by spreading slurries of Fe nanoparticle-loaded carbon materials, NMP and polyvinylidene fluoride (SOLEF of Solvay Chemicals, Belgium) as a binder (10 wt\%) on a glassy carbon electrode followed by drying under a halogen lamp. The amount of active material deposited on the glassy carbon electrode was $1.5 \pm 0.01 \mathrm{mg}$, which was weight difference of the glassy carbon electrode before and after loading the active material. Cyclic voltammetry was performed in $1.0 \mathrm{M}$ KOH electrolyte (Wako Pure Chemical Co., Ltd., Japan) under nitrogen atmosphere using a potentiostat (HZ-3000 automatic polarization system, Hokuto Denko, Japan) for a conventional three-electrode system. The potentials were measured against a $\mathrm{Ag} / \mathrm{AgCl}$ reference electrode with a saturated $\mathrm{KCl}$ solution (RE-1C, BAS Inc., Japan). The scan rate was $10 \mathrm{mV} / \mathrm{sec}$. Impedance measurements were performed using a frequency response analyzer (Model 5080, NF Electric Instruments, Japan) and the potentiostat by applying a low-amplitude alternating voltage of $5 \mathrm{mV}$ at open circuit potential $(-0.2 \mathrm{~V})$ in the frequency range of $10 \mathrm{kHz} \sim 100 \mathrm{mHz}$.

\section{Results and Discussion}

\subsection{Characterization of Fe nanoparticles on TCNF and CCNT}

Fig. 1 shows TEM images of TCNF and CCNT before and after graphitization. As shown in Fig. 1(a), the pristine TCNF shows a periodic structure of funnel-type structural units $[31,32]$ revealed by the fractures between structural units as indicated with the arrows (See also the zoomed image.). The funnel-type units, whose walls are tilted by $\sim 5^{\circ}$ to the fiber axis, repeat at a periodicity of approximately $20 \sim 30 \mathrm{~nm}$. Graphitization of TCNF at $2800^{\circ} \mathrm{C}$ enhances the periodic structure as shown in Fig. 1(b). Specifically, the graphitized TCNF consists of funnel-type units joining consecutively at a period of $\sim 10 \mathrm{~nm}$ to clearly show the presence of fractures between the structural units. Note that the walls of the nanotube segments in graphitized TCNF are tilted by $\sim 8^{\circ}$ to the fiber axis. In pristine CCNT, on the other hand, there is a bamboo-like joint (indicated by arrows) (Fig. 1(c)). However, such a joint is not observed at all after graphitization of CCNT (Fig. 1(d)). Therefore, the fractures observed in this report are unique only with TCNF, not with CCNF. 

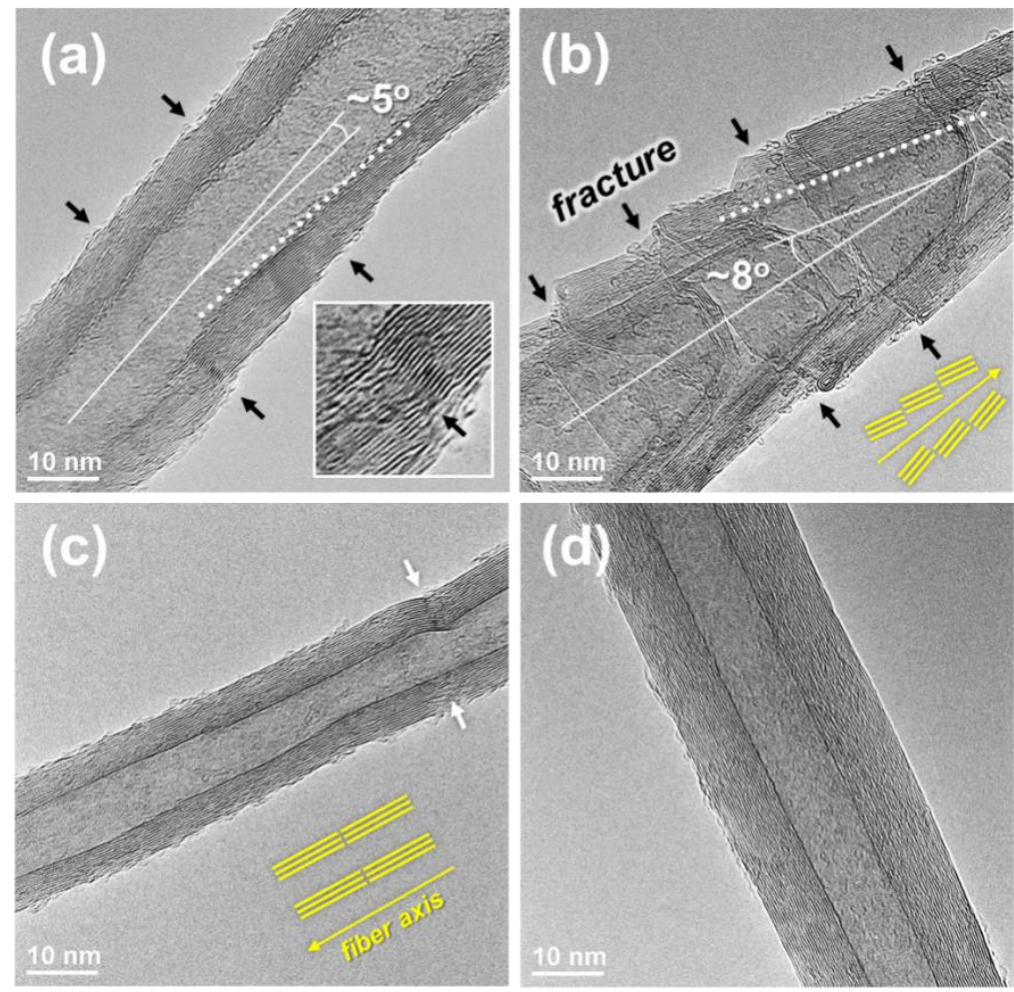

Fig. 1. TEM images of (a) pristine TCNF, (b) graphitized TCNF, (c) pristine CCNT and (d) graphitized CCNT. The arrows indicate fractures in TCNF and a joint in CCNT. The inset is a zoomed image of a fracture on pristine TCNF.

The exterior surfaces of TCNF and CCNT were also examined with STM to provide three-dimensional topological information. The surfaces of pristine TCNF and CCNT appear smooth as shown in Figs. 2(a) and (c); however, the images of a higher magnification (the insets of Figs. 2(a) and (c)) clearly demonstrate the existence of the fractures and bamboo-like joints on the surfaces of pristine TCNF and CCNT, respectively. Graphitization brought dramatic changes in the topographies of TCNF and CCNT. Specifically, the fractures become much more enhanced on graphitized TCNF (Fig. 2(b)), while the joint is rarely observable on graphitized CCNT (Fig. 2(d)). Furthermore, the STM image of graphitized TCNF reveals that the interval of the fracture is roughly around $10 \mathrm{~nm}$, and that the structural basic unit on TCNF are carbon nano-plate, not carbon nano-rod [31, 32]. Thus, the results of STM are quite consistent with those of TEM. 

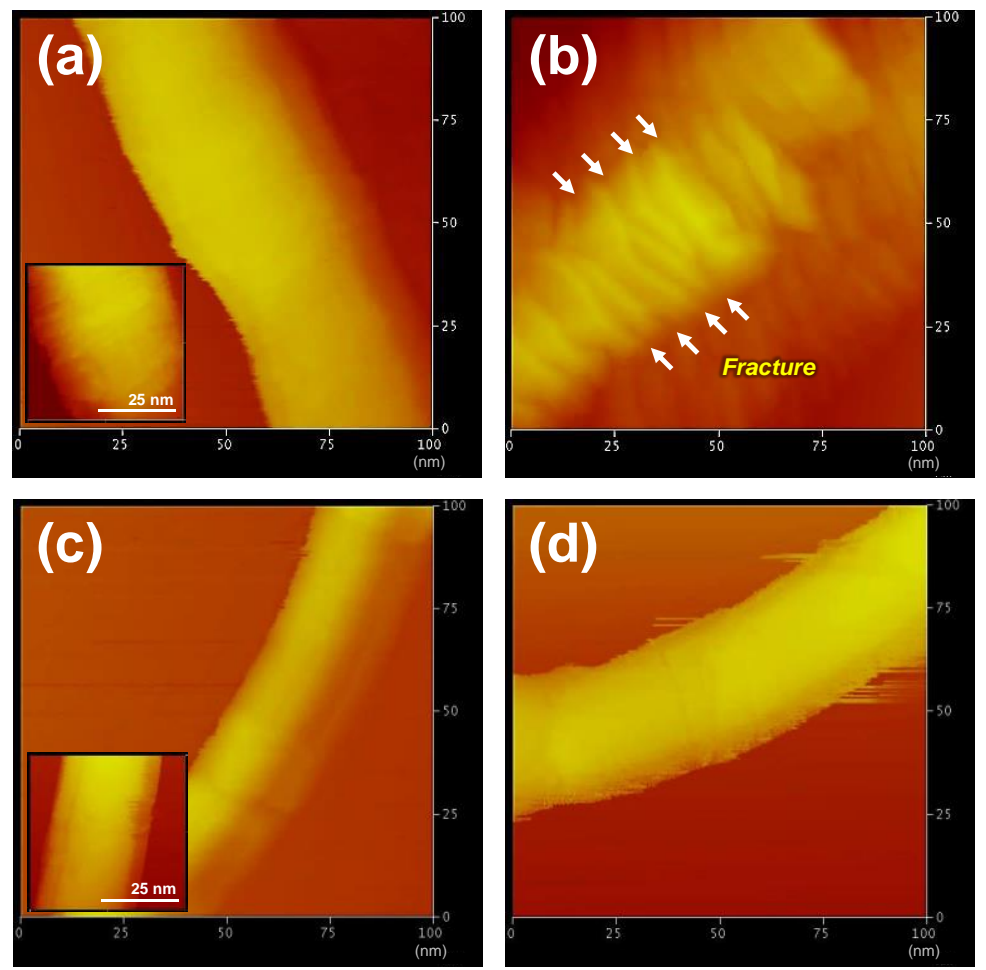

Fig. 2. STM images of (a) pristine and (b) graphitized TCNF, and (c) pristine and (d) graphitized CCNT. The insets are higher magnifications of each STM images. The arrows indicate the fractures of graphitized TCNF.

Fig. 3 shows TEM images of Fe nanoparticles loaded on pristine TCNF and CCNT. Fig. 3(a) obviously demonstrates that the majority of Fe nanoparticles (5 $10 \mathrm{~nm}$ in diameter) are in hollow TCNF tubes (Fig. 3(a)). Contrastingly, the individuals or aggregates of Fe nanoparticles of relatively larger sizes are scattered on the surfaces of CCNT (Fig. 3(b)). Indeed, there is no Fe nanoparticle in the tubes of CCNT. The only difference between TCNF and CCNT is that the fractures are on TCNF, while the bamboo-like joints are on CCNT. Accordingly, the fractures between the structural basic units of TCNF are associated with the residence of the Fe nanoparticles in TCNF tubes: the fractures play a role of gate (nano-gate) in penetration of Fe precursor ions into hollow TCNF tubes during impregnation step. The entrained Fe precursor ions are reduced to Fe nanoparticles during thermal reduction step. 

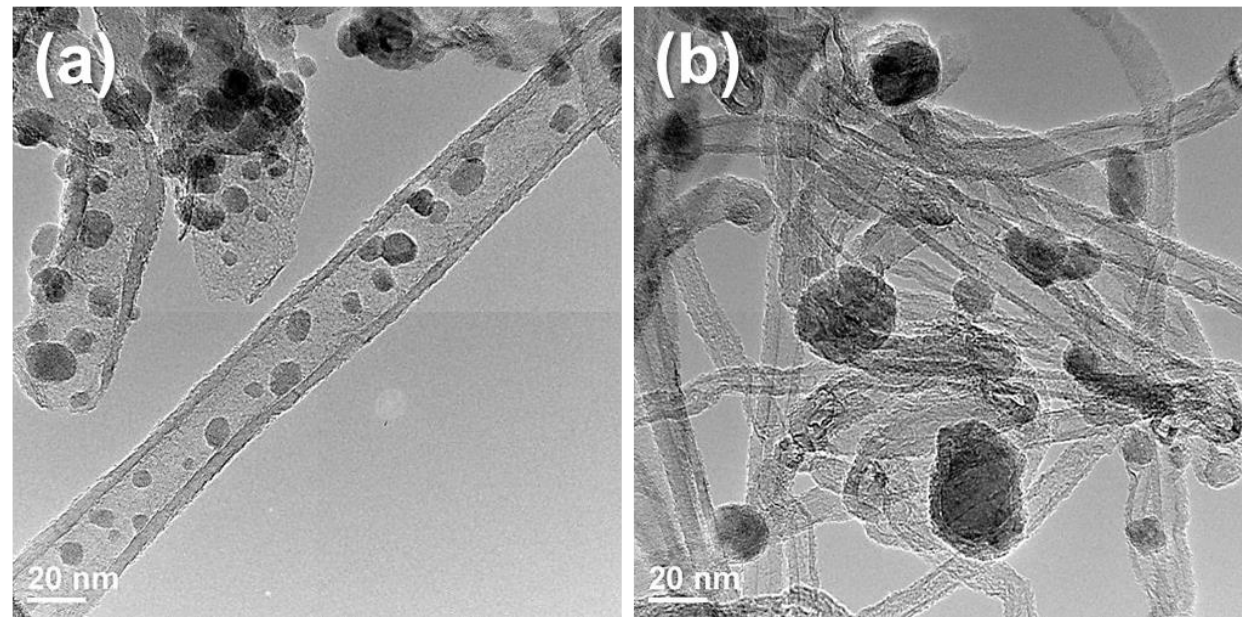

Fig. 3. Typical TEM images of Fe-loaded (a) TCNF and (b) CCNT.

Fig. 4 shows weight variations of pristine TCNF and CCNT without and with loaded Fe nanoparticles during TGA analysis under air atmosphere. The loaded amount of Fe was $\sim 5$ wt $\%$.

Fe-loaded TCNF

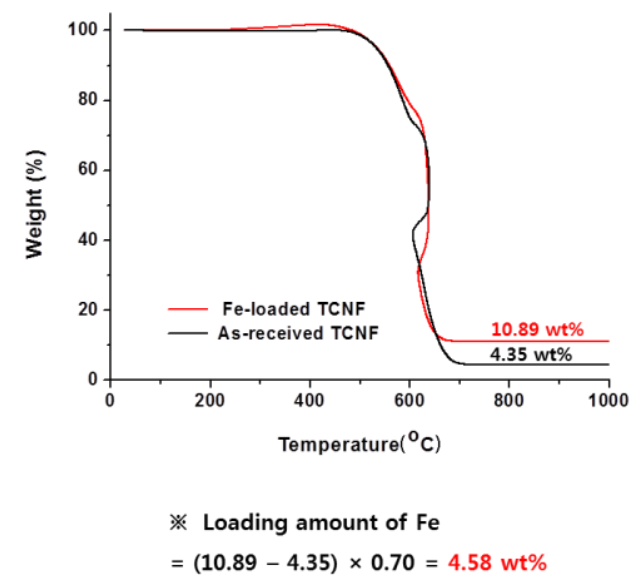

Fe-loaded CCNT

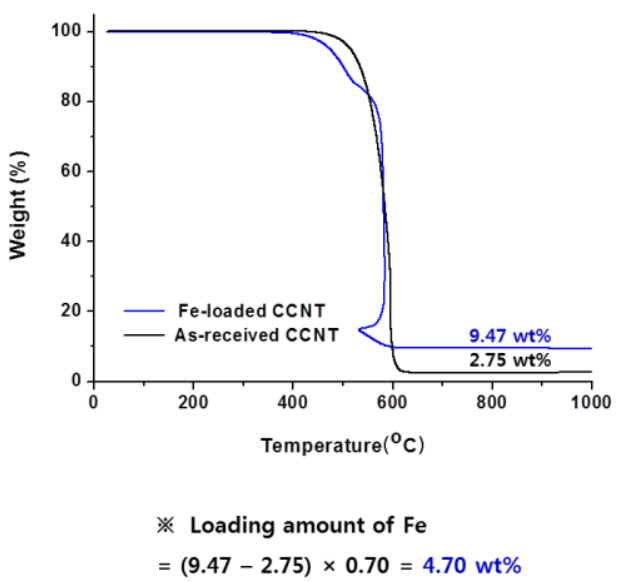

Fig. 4. TGA analyses of (a) TCNF and (b) CCNT without and with Fe nanoparticles. 0.70 was derived by $2 \times \mathrm{Fe} / \mathrm{Fe}_{2} \mathrm{O}_{3}$ assuming that the remained iron oxide is $\mathrm{Fe}_{2} \mathrm{O}_{3}$.

Crystallographic phases of Fe nanoparticles loaded on TCNF and CCNT were scrutinized using XRD as shown in Fig. 5. The impregnated nanoparticles on both carbon materials were spinel $\gamma$ type $\mathrm{Fe}_{2} \mathrm{O}_{3}$ (PDF file No. 00-039-1346) with a small amount of metallic Fe (PDF file No. 01-071-3763) [33]. No $\mathrm{Fe}_{3} \mathrm{O}_{4}$ was found in both samples. Certainly, metallic nanoparticles were oxidized during storage in air for further investigations despite the reduction process of heat treatment at $500^{\circ} \mathrm{C}$ for $1 \mathrm{~h}$ under an $\mathrm{Ar}$ atmosphere. Although the 
crystallographic phase of the pristine Fe nanoparticles on TCNF and CCNT is verified to be $\mathrm{Fe}_{2} \mathrm{O}_{3}$, the term "Fe nanoparticle" is used collectively to designate metallic Fe nanoparticle and Fe oxide nanoparticles unless specified.

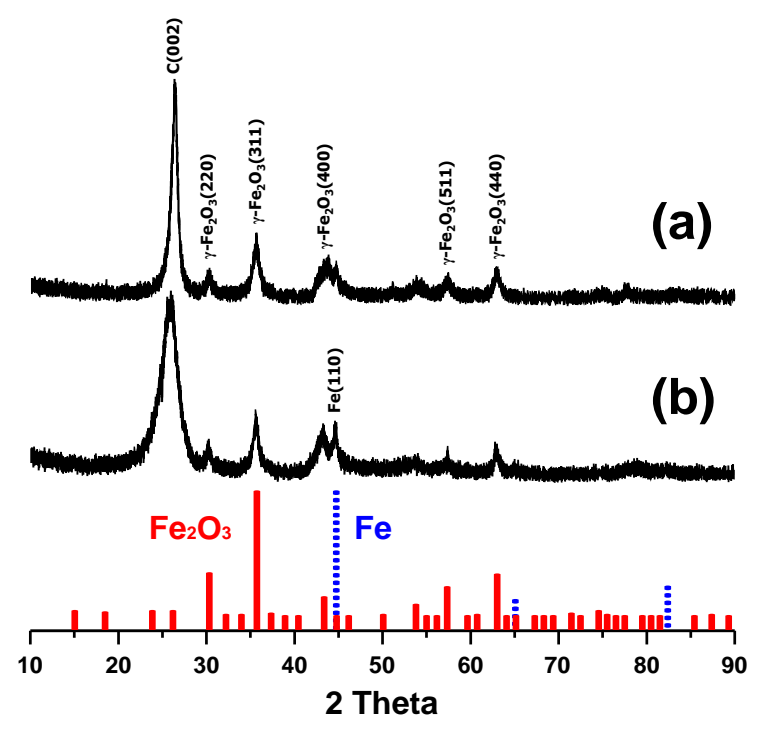

Fig. 5. XRD patterns of Fe-loaded (a) TCNF and (b) CCNT.

\subsection{Electrochemical properties of Fe nanoparticles on TCNF and CCNT}

Fig. 6 shows cyclic voltammograms of Fe nanoparticles loaded on TCNF and CCNT in 1.0 $\mathrm{M} \mathrm{KOH}$. In the voltammograms observed in the initial cathodic scan on both carbon materials (the dashed lines in Fig. 6), there are two reduction processes peaking roughly at $1.1 \mathrm{~V}$ and starting at $-1.15 \mathrm{~V}$. In the following second scan, only one redox couple is observed with oxidation and reduction peak potentials approximately at -0.7 and $-1.1 \mathrm{~V}$, respectively. A clear difference in electrochemical reduction of $\mathrm{Fe}$ nanoparticles is that two peaks are observed in the first cycle, while only one is discernible at $-1.1 \mathrm{~V}$ in the second cycle. In the continued cycles, the reduction peak at $-1.1 \mathrm{~V}$ is persistent. Because the reduction charge starting $-1.15 \mathrm{~V}$ is similar to the one in the second cycle, a Fe species would be responsible for the reduction process starting $-1.15 \mathrm{~V}$ in the initial cathodic scan. Then, the pristine $\mathrm{Fe}$ oxide ( $\gamma$ type $\mathrm{Fe}_{2} \mathrm{O}_{3}$ as demonstrated in Fig. 5) formed in air may differ from a $\mathrm{Fe}$ oxide produced by electrochemical oxidation in voltammetric cycles. This interpretation is applicable to the first and second voltammetric scans of TCNF and CCNT, despite a slight difference in the shape of the redox curves. However, as the number of voltammetric cycles increases, the evolution of voltammogram on TCNF and CCNT become distinguishing from each other. Specifically, the redox peaks of Fe nanoparticles on TCNF (Fig. 6(a)) become sharper as signaled by decreases in peak width at half maximum from $0.12 \mathrm{~V}\left(1^{\text {st }}\right.$ cycle) to 
$0.08 \mathrm{~V}\left(100^{\text {th }}\right.$ cycle $)$ for the anodic peak and $0.09 \mathrm{~V}\left(2^{\text {nd }}\right.$ cycle $)$ to $0.06 \mathrm{~V}\left(100^{\text {th }}\right.$ cycle $)$ for the cathodic peak. Furthermore, the oxidation/reduction peak potentials shifts in cathodic and anodic directions, respectively, to decrease the potential difference from $0.38 \mathrm{~V}$ ( $1^{\text {st }}$ cycle) to $0.33 \mathrm{~V}\left(100^{\text {th }}\right.$ cycle). On the other hand, in the voltammograms of CCNT (Fig. 6(b)) there are two redox couples: one is a redox couple nearly identical to that observed on TCNF in terms of peak potential (-0.73 and $-1.06 \mathrm{~V}$ for anodic and cathodic peaks, respectively) and their potential difference $(0.33 \mathrm{~V})$, and the other is a new redox couple appears at the shoulders of the previously described redox couple to show a much wider potential difference of $0.51 \mathrm{~V}$ in the second cycle. As voltammeric cycle continues, moreover, the charge of the new redox couple keeps increasing at the expense of the other redox couple of less peak separation, and its oxidation/reduction potentials shifts progressively in the anodic and cathodic directions, respectively. Additionally, it is important to recognize that the charge involved in the redox process of Fe nanoparticles on TCNF is larger than that on CCNT by a factor of two (Note the different current density scale of Figs. 6(a) and (b).). Therefore, the electrochemical characteristics of Fe nanoparticles in the hollow TCNF tubes are more favorable than those on CCNT in terms of overpotential as well as charges involved in the redox reaction, despite an identical amount of loaded Fe nanoparticles ( $5 \mathrm{wt} \%)$.
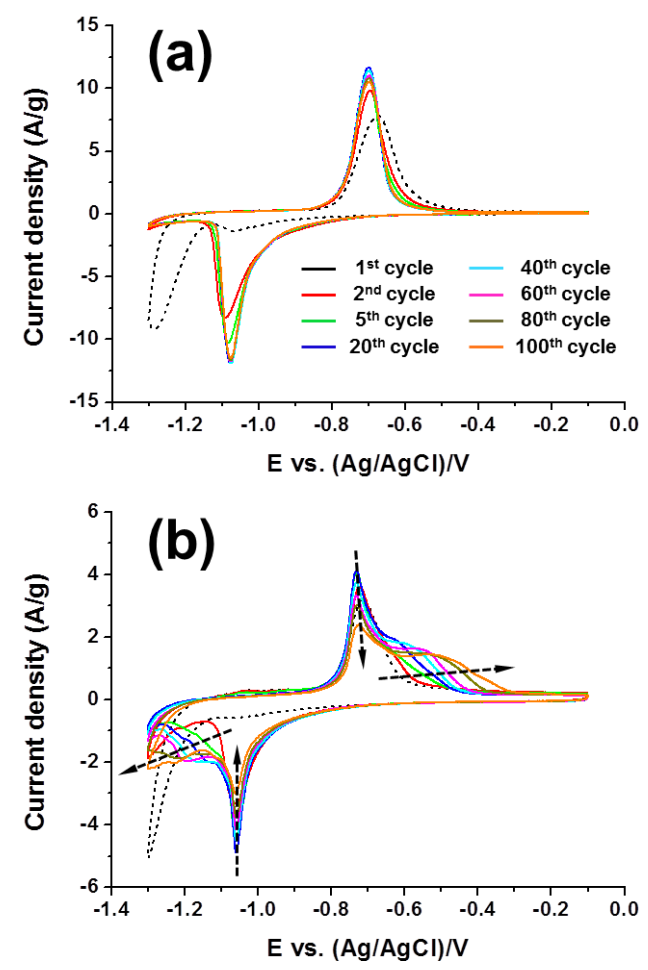

Fig. 6. Cyclic voltammograms of Fe-loaded (a) TCNF and (b) CCNT in 1.0 M KOH solution. Note the difference in current density scale of (a) and (b). Scan rate: $10 \mathrm{mV} / \mathrm{sec}$. 


\subsection{Evolution of Fe nanoparticles on TCNF and CCNT during electrochemical treatment}

Fig. 7 show TEM images of Fe nanoparticles on TCNF and CCNT modified after the $100^{\text {th }}$ voltammetric cycle in 1.0 M KOH. During the voltammetric cycles between -0.1 and $-1.3 \mathrm{~V}$, $\mathrm{Fe}$ nanoparticles must experience sequential repetition of a dissolution process of metallic $\mathrm{Fe}$ nanoparticles to soluble Fe species and a re-deposition process of the soluble Fe species onto the surfaces of nearby $\mathrm{Fe}$ nanoparticles. Therefore, the shapes of $\mathrm{Fe}$ nanoparticles are expected to be modified significantly. Indeed, the spherical Fe nanoparticles in TCNF tubes before the electrochemical treatment (Fig. 3(a)) are modified to elongated nanoparticles (Fig. 7(a)). In contrast, the Fe nanoparticles on CCNT change to large dendrites (Fig. 7(b)), which are not desirable. Growth of the elongated Fe nanoparticles in TCNF tubes would be due to confinement of the soluble species generated during the repeated dissolution/re-deposition processes in TCNF tubes. Fe precursor ions took as long as 3 days to penetrate into TCNF tubes through the nano-gates during impregnation step, and the time scale in a voltammetric scan (one voltammetric cycle took $4 \mathrm{~min}$ ) is too short for the soluble species to diffuse out of TCNF tubes through the nano-gates. Thus, all electrochemical processes on TCNF (i.e., dissolution to soluble species and re-deposition of the soluble species) occur in the confined spaces of TCNF tubes. When the Fe nanoparticles dissolve, and when the soluble species redeposit on nearby Fe seed nanoparticles, the soluble species diffuse along the tubes in both directions (one-dimensional diffusion). On CCNT, on the other hand, the soluble species diffuses three-dimensionally during the dissolution and re-deposition processes. However, the absence of confined spaces in CCNT would lead to dendrite formation. Because the soluble species of nanoparticles on CCNT are able to diffuse in all directions, only large dendrites will survive after a large number of voltammetric cycles. Indeed, spherical Fe nanoparticles are not observed around the dendrites after $100^{\text {th }}$ cycles as shown in Fig. 7(b). 

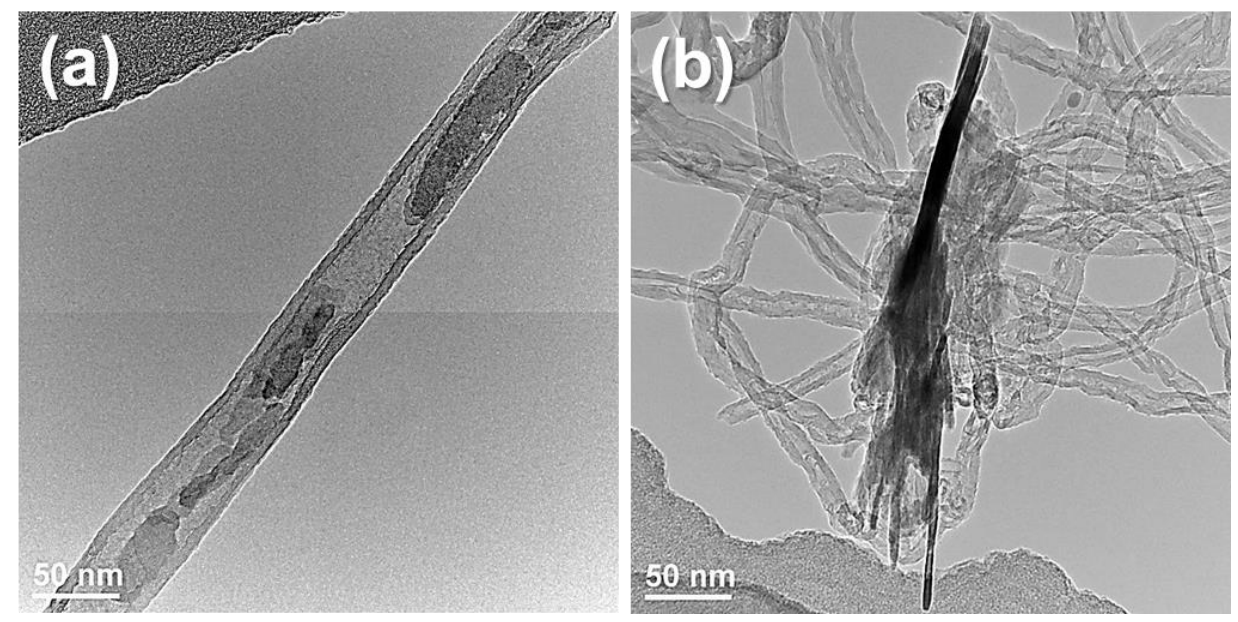

Fig. 7. TEM images of Fe-loaded (a) TCNF and (b) CCNT after $100^{\text {th }}$ voltammetric cycles in $1 \mathrm{M} \mathrm{KOH}$ solution.

Figs. 8 and 9 show the typical results of TEM-EDX analysis of Fe nanoparticles on TCNF and CCNT after the $100^{\text {th }}$ cycle. The elongated features in TCNF are mainly composed of Fe with an insignificant amount of oxygen (Fig. 8). On the other hand, the components of the thick object on CCNT in Fig. 9 are Fe and oxygen. Thus, a prolonged voltammetric treatment results in Fe nanoparticles of different chemical compositions depending on whether they are in confined space or not. In other words, Fe nanoparticles evolve to oxygen-lean elongated $\mathrm{Fe}$ nanoparticles in hollow TCNF tubes, while they grow to oxygen-rich Fe oxide dendrite nanoparticles on the surfaces of CCNT.
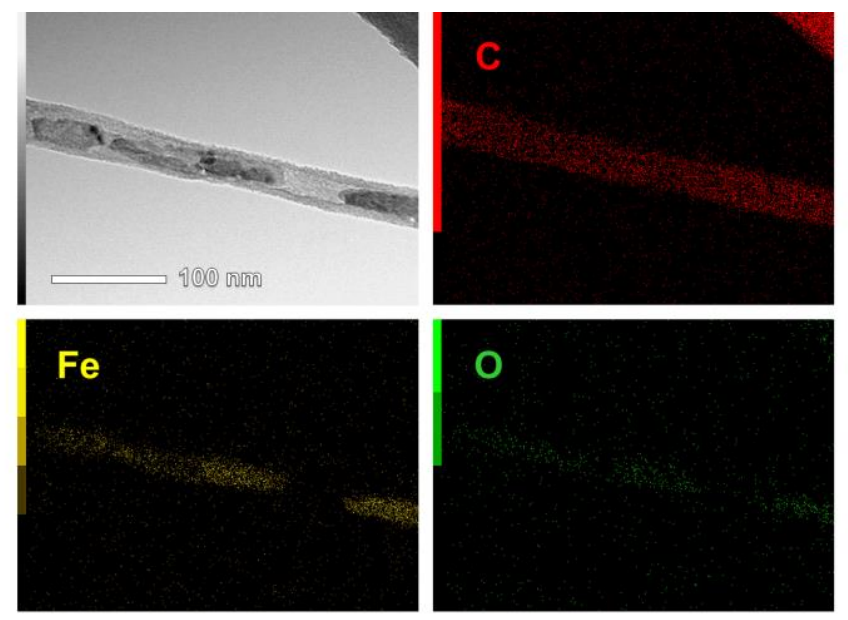

Fig. 8. TEM-EDX images of Fe nanoparticles in TCNF after the $100^{\text {th }}$ cycle in $1.0 \mathrm{M} \mathrm{KOH}$. 

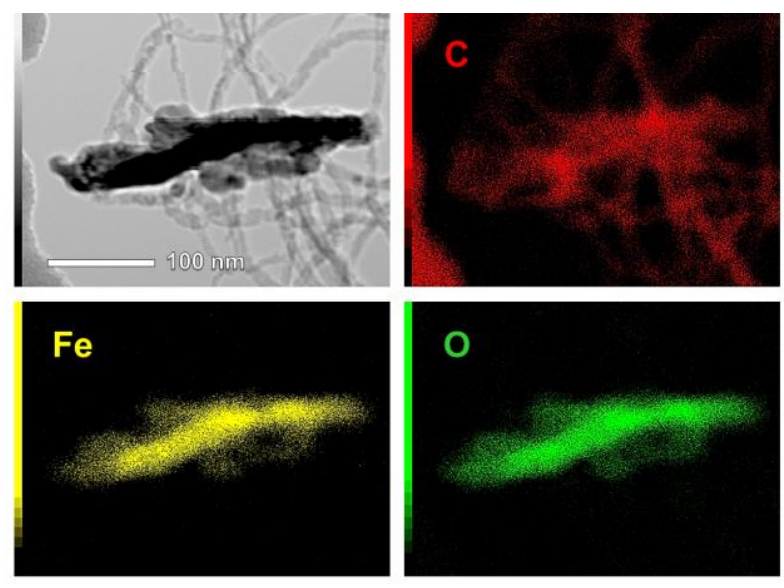

Fig. 9. TEM-EDX images of a Fe oxide dendrite on CCNT after the $100^{\text {th }}$ cycle in $1.0 \mathrm{M}$ $\mathrm{KOH}$.

Fig. 10 shows the Nyquist plots of the dissolution processes of Fe nanoparticles on TCNF and CCNT in $1.0 \mathrm{M} \mathrm{KOH}$ at open circuit potential $(\sim-0.2 \mathrm{~V})$. The aim for the choice of the particular potential is to investigate the mass transfer which is a clue to understanding the difference in evolution of Fe particles on TCNF and CCNT after a long-term voltammeric cycle. On both of TCNF and CCNT, the charge transfer resistances at high frequency are extremely small and similar, obviously indicating that the dissolution reaction is fast at $-0.2 \mathrm{~V}$ [34-37]. However, the mass transfer resistances at low frequency relating diffusion of the soluble species greatly depended on carbon substrate [34-37]. Specifically, from the plot of absolute value of impedance $\left(\mathrm{Z}^{\prime \prime}\right.$, ohms $(\Omega)$ ) on the operating frequency (Fig. 11), the impedance resistance of TCNF $(\sim 3.5 \Omega$, at $10 \mathrm{~Hz})$ is fairly constant independently from the number of voltammetric cycle, while that of CCNT increases from $\sim 3.5$ to $\sim 9.5 \Omega$, at $10 \mathrm{~Hz}$. The observed impedance values relating with mass transfer resistance supports that the diffusion of the soluble species in the TCNF tubes is much faster than in the electrochemical medium surrounding CCNT. The specific reason for the faster diffusion in the tube is the increased concentration gradient of soluble species because of the confined nanometer-sized spaces. In turn, the faster diffusion in TCNF may increase the amount of Fe nanoparticles participating in the dissolution and re-deposition process to increase the charge under voltammetric curves in Fig. 6. On the other hand, the variation in mass transfer resistance of Fe nanoparticles on CCNF depends on the number of voltammetric cycles, which suggests that the diffusion rate of soluble species decreases along with dendrite growth during a longterm electrochemical treatment. 


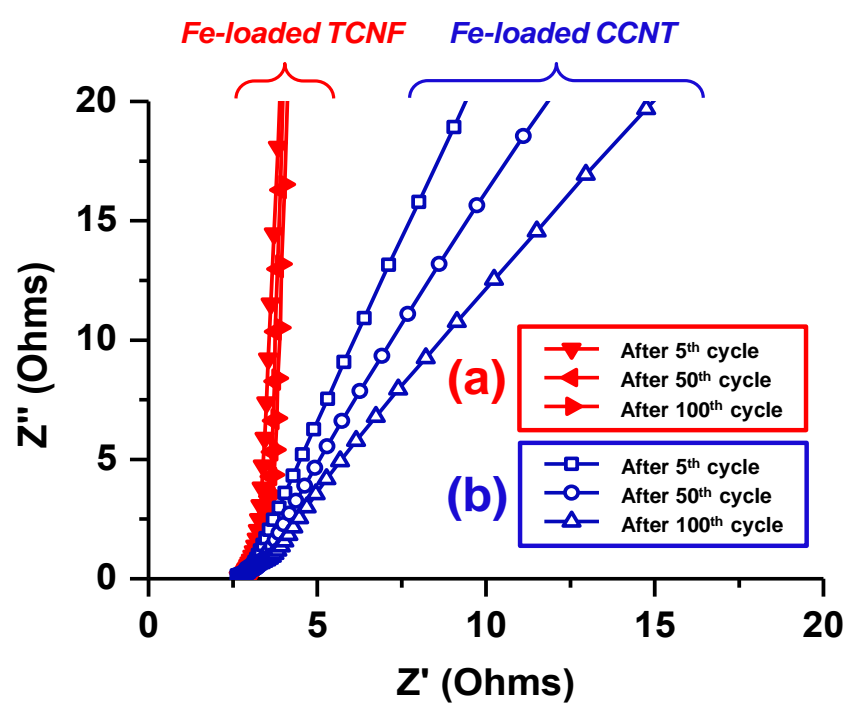

Fig. 10. The Nyquist plots of Fe-loaded (a) TCNF and (b) CCNT after the $5^{\text {th }}, 50^{\text {th }}$ and $100^{\text {th }}$ cycling in $1.0 \mathrm{M} \mathrm{KOH}$ at open circuit potential $(-0.2 \mathrm{~V})$.

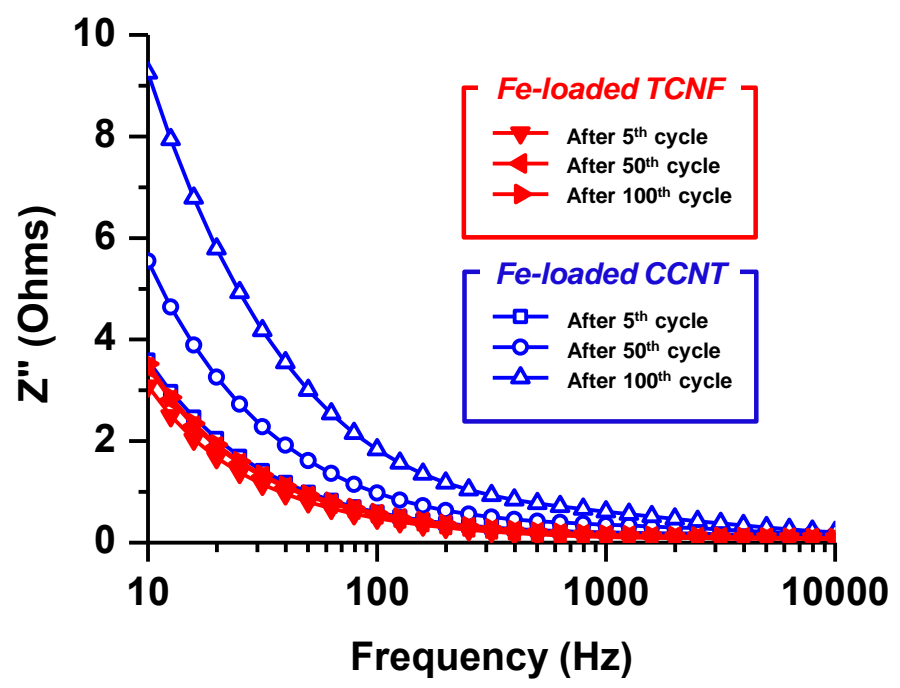

Fig. 11. Absolute value of impedance $\left(Z^{\prime \prime}\right.$, ohms $(\Omega)$ ) for the dissolution process of Fe nanoparticles on TCNF and CCNT in $1.0 \mathrm{M} \mathrm{KOH}$ solution at open circuit potential $(\sim-0.2 \mathrm{~V})$ as a function of frequency in impedance.

The appearance of the new redox process on CCNT (Fig. 6(b)) should be addressed in detail here. The new redox process of Fe nanoparticles on CCNT is certainly related to growth of oxygen-rich Fe oxide dendrites evolved from spherical nanoparticles as voltammetric cycle continues. Because the anodic and cathodic potentials of the new redox process are higher and lower than those on TCNF, respectively, and because the potential difference between them keeps increasing, the overpotential of the new redox process certainly gradually become larger. The specific dendrite grown on CCNT may be less electronically conductive or 
inherently less reactive especially in dissolution than the Fe nanoparticle in TCNF tubes. In any cases, a higher overpotential is needed to maintain the dissolution of dendrite and redeposition to dendrite. At the potential for impedance investigation $(-0.2 \mathrm{~V})$, the threedimensional concentration gradient of the soluble Fe species from dendrites would be much lower due to the overpotential for dissolution of dendrite than one-dimensional concentration gradient from spherical nanoparticles of small overpotential. Thus, the higher overpotential of dendrite dissolution (leading to a lower concentration gradient of soluble Fe species) and higher three-dimensional mass transfer resistance leads to a less amount of Fe involved in charging/discharging process. In a reverse way, the lower overpotential of oxygen-lean Fe nanoparticles and the low one-dimensional mass transfer resistance of soluble Fe species account for the high efficiency of Fe nanoparticles in TCNF regarding Fe-air battery system.

\section{Conclusions}

This work presented a fundamental reason for the higher efficiency of Fe nanoparticles loaded on TCNF for metal-air battery previously reported by us $[24,26,29,30]$. The Fe precursor ions penetrated into the TCNF tubes through the fractures (or nano-gates) between the structural units of TCNF, which are absent in CCNT. During electrochemical dissolution and re-deposition processes of Fe nanoparticles, the Fe nanoparticles evolved to elongated oxygen-lean $\mathrm{Fe}$ nanoparticles in the confined space of TCNF tubes and to oxygen-rich $\mathrm{Fe}$ oxide dendrites on CCNT. The dendrites on CCNT needed a higher overpotential for a dissolution and re-deposition process as verified by voltammetric study. As a consequence, the mass transfer of soluble Fe species was faster in the confined space of TCNF tubes than in the open space between CCNT. The higher efficiency of Fe nanoparticles loaded on TCNF for metal-air battery was originated from the low overpotential of elongated oxygen-lean $\mathrm{Fe}$ nanoparticles and the fast one-dimensional mass transfer of soluble Fe species in hollow TCNF tubes.

The fundamental issue concerning Fe nanoparticles loaded on TCNF presented in this work is a certain break-through toward practical metal-air battery. In this regard, a research work for more practical Fe-air battery utilizing the concept of penetration of metal precursor ions into confined spaces is in progress in our group.

\section{Acknowledgements}

This work was supported by a grant from the ALCA project in Japan Science and Technology Agency (JST) and the Global Centre of Excellence (G-COE) of Novel Carbon 
Resource Sciences in Kyushu University. The authors thank JST and Kyushu University for financial assistance. CKR thanks Chungnam National University for a financial support (project number: 2014-0696-01).

\section{References}

[1] Girishkumar G, McCloskey B, Luntz AC, Swanson S, Wilcke W. Lithium-air battery: promise and challenges. J Phys Chem Lett 2010; 1(14):2193-203.

[2] Jung H-G, Hassoun J, Park J-B, Sun Y-K, Scrosati B. An improved high-performance lithium-air battery. Nat Chem 2012; 4(7):579-85.

[3] Kubo D, Tadanaga K, Hayashi A, Tatsumisago M. Multifunctional inorganic electrode materials for high-performance rechargeable metal-air batteries. J Mater Chem A 2013; 1(23):6804-9.

[4] Yuasa M, Imamura H, Nishida M, Kida T, Shimanoe K. Preparation of nano-LaNiO 3 support electrode for rechargeable metal-air batteries. Electrochem Commun 2012; 24(0):50-2 [5] Rahman MA, Wang X, Wen C. High energy density metal-air batteries: A Review. J Electrochem Soc 2013; 160(10):A1759-A71.

[6] Lee J-S, Tai Kim S, Cao R, Choi N-S, Liu M, Lee KT, et al. Metal-air batteries: metal-air batteries with high energy density: Li-Air versus Zn-Air. Adv Energy Mater 2011; $1(1): 34-50$.

[7] Chakkaravarthy C, Waheed AKA, Udupa HVK. Zinc-air alkaline batteries - A review. J Power Sources 1981; 6(3):203-28.

[8] Kitamura H, Zhao L, Hang BT, Okada S, Yamaki J. Effect of binder materials on cycling performance of $\mathrm{Fe}_{2} \mathrm{O}_{3}$ electrodes in alkaline solution. J Power Sources 2012; 208(0):391-6.

[9] Ma M, Tu JP, Yuan YF, Wang XL, Li KF, Mao F, et al. Electrochemical performance of $\mathrm{ZnO}$ nanoplates as anode materials for $\mathrm{Ni} / \mathrm{Zn}$ secondary batteries. J Power Sources 2008; 179(1):395-400.

[10] Lee CW, Sathiyanarayanan K, Eom SW, Yun MS. Novel alloys to improve the electrochemical behavior of zinc anodes for zinc/air battery. J Power Sources 2006; 160(2):1436-41.

[11] Lee CW, Sathiyanarayanan K, Eom SW, Kim HS, Yun MS. Novel electrochemical behavior of zinc anodes in zinc/air batteries in the presence of additives. J Power Sources 2006; 159(2):1474-7. 
[12] Zhang C, Wang JM, Zhang L, Zhang JQ, Cao CN. Study of the performance of secondary alkaline pasted zinc electrodes. J Appl Electrochem 2001; 31(9):1049-54.

[13] Cho Y-D, Fey GT-K. Surface treatment of zinc anodes to improve discharge capacity and suppress hydrogen gas evolution. J Power Sources 2008; 184(2):610-6.

[14] Yano M, Fujitani S, Nishio K, Akai Y, Kurimura M. Effect of additives in zinc alloy powder on suppressing hydrogen evolution. J Power Sources 1998; 74(1):129-34.

[15] Zhu L, Zhang H, Li W, Liu H. New modification procedure of zinc powder in neodymium nitrate solution for improving the electrochemical properties of alkaline zinc electrodes. J Phys Chem Solids 2009; 70(1):45-54.

[16] Ein-Eli Y, Auinat M, Starosvetsky D. Electrochemical and surface studies of zinc in alkaline solutions containing organic corrosion inhibitors. J Power Sources 2003; 114(2):3307.

[17] Kim K, Cho Y-H, Eom SW, Kim H-S, Yeum JH. Anions of organic acids as gas suppressants in zinc-air batteries. Mater Res Bull 2010; 45(3):262-4.

[18] Blurton KF, Sammells AF. Metal/air batteries: Their status and potential - A review. J Power Sources 1979; 4(4):263-79.

[19] Palacin MR. Recent advances in rechargeable battery materials: a chemist's perspective. Chem Soc Rev 2009; 38(9):2565-75.

[20] Yang C-C, Lin S-J. Alkaline composite PEO-PVA-glass-fibre-mat polymer electrolyte for Zn-air battery. J Power Sources 2002; 112(2):497-503.

[21] Xu JJ, Ye H, Huang J. Novel zinc ion conducting polymer gel electrolytes based on ionic liquids. Electrochem Commun 2005; 7(12):1309-17.

[22] Simons TJ, Torriero AAJ, Howlett PC, MacFarlane DR, Forsyth M. High current density, efficient cycling of $\mathrm{Zn}^{2+}$ in 1-ethyl-3-methylimidazolium dicyanamide ionic liquid: The effect of $\mathrm{Zn}^{2+}$ salt and water concentration. Electrochem Commun 2012; 18(0):119-22.

[23] Hang BT, Watanabe T, Eashira M, Watanabe I, Okada S, Yamaki J. Comparative study of $\mathrm{Fe}_{2} \mathrm{O}_{3}$-nanoloaded carbon and $\mathrm{Fe}_{2} \mathrm{O}_{3}$-nano/carbon mixed composites for iron-air battery anodes. Electrochem Solid-State Lett 2005; 8(9):A476-A80.

[24] Hang BT, Eashira M, Watanabe I, Okada S, Yamaki J, Yoon S-H, et al. The effect of carbon species on the properties of $\mathrm{Fe} / \mathrm{C}$ composite for metal-air battery anode. $\mathrm{J}$ Power Sources 2005; 143(1-2):256-64.

[25] Hang BT, Hayashi H, Yoon S-H, Okada S, Yamaki J. $\mathrm{Fe}_{2} \mathrm{O}_{3}$-filled carbon nanotubes as a negative electrode for an Fe-air battery. J Power Sources 2008; 178(1):393-401. 
[26] Hang BT, Watanabe T, Eashira M, Okada S, Yamaki J, Hata S, et al. The electrochemical properties of $\mathrm{Fe}_{2} \mathrm{O}_{3}$-loaded carbon electrodes for iron-air battery anodes. $\mathrm{J}$ Power Sources 2005; 150(0):261-71.

[27] Hang BT, Thang DH, Nga NT, Minh PTL, Kobayashi E. Nanoparticle $\mathrm{Fe}_{2} \mathrm{O}_{3}$-loaded carbon nanofibers as iron-air battery anodes. J Electrochem Soc 2013; 160(9):A1442-A5.

[28] Ito A, Zhao L, Okada S, Yamaki J. Synthesis of nano- $\mathrm{Fe}_{3} \mathrm{O}_{4}$-loaded tubular carbon nanofibers and their application as negative electrodes for Fe/air batteries. J Power Sources 2011; 196(19):8154-9.

[29] Hang BT, Yoon S-H, Okada S, Yamaki J. Effect of metal-sulfide additives on electrochemical properties of nano-sized $\mathrm{Fe}_{2} \mathrm{O}_{3}$-loaded carbon for Fe/air battery anodes. $\mathrm{J}$ Power Sources 2007; 168(2):522-32.

[30] Hang BT, Watanabe T, Egashira M, Watanabe I, Okada S, Yamaki J. The effect of additives on the electrochemical properties of $\mathrm{Fe} / \mathrm{C}$ composite for Fe/air battery anode. $\mathrm{J}$ Power Sources 2006; 155(2):461-9.

[31] Yoon S-H, Lim S, Hong S-H, Qiao W, Whitehurst DD, Mochida I, et al. A conceptual model for the structure of catalytically grown carbon nano-fibers. Carbon 2005; 43(9):182838.

[32] Long D, An B, Yokogawa K, Ling L, Miyawaki J, Mochida I, et al. Structural units and their periodicity in carbon nanotubes. Small 2010; 6(22):2526-9.

[33] Li J, Lin YS. Facile synthesis of ordered mesoporous silica with high $\gamma-\mathrm{Fe}_{2} \mathrm{O}_{3}$ loading via sol-gel process. J Mater Sci 2008; 43(18):6359-65.

[34] Wen ZB, Qu QT, Gao Q, Zheng XW, Hu ZH, Wu YP, et al. An activated carbon with high capacitance from carbonization of a resorcinol-formaldehyde resin. Electrochem Commun 2009; 11(3):715-8.

[35] Tanaka S, Doi A, Matsui T, Miyake Y. Mass transport and electrolyte accessibility through hexagonally ordered channels of self-assembled mesoporous carbons. J Power Sources 2013; 228(0):24-31.

[36] Wei T-C, Hillhouse HW. Mass transport and electrode accessibility through periodic self-assembled nanoporous silica thin films. Langmuir 2007; 23(10):5689-99.

[37] Wang L, Zhou Y, Qiu J. Influence of pore structures on the electrochemical performance of asphaltene-based ordered mesoporous carbons. Microporous Mesoporous Mater 2013; 174(0):67-73. 